\title{
DETERMINACIÓN DEL PH SALIVAL EN PACIENTES ADULTOS CON DIABETES MELLITUS COMPENSADOS
}

\author{
DETERMINATION OF SALIVAL PH IN ADULT PATIENTS WITH \\ COMPENSATED DIABETES MELLITUS
}

\author{
María Dayan Avellaneda Lopez 1,a
}

\begin{abstract}
RESUMEN
Objetivo. Determinar el pH salival en pacientes adultos con diabetes mellitus (DM), compensados atendidas en el centro de salud público de Callao. Materiales y métodos. Fue estudio analítico, observacional, casos y controles. Muestra de 50 personas con rango en edad de 20 a 60 años. Se realizó inducción general sobre el estudio, para obtener el consentimiento informado. Se les indicó que contengan la saliva que se va acumulando en la boca por unos minutos y luego, en cada minuto, la saliva se iba vertiendo en un embudo de vidrio conectado a una probeta desinfectada y limpia, graduada en $10 \mathrm{ml}$, esta actividad se realizó durante un minuto. Una vez recogida la muestra se procedió a colocarla en un lugar fresco y seguro para dejar que pueda reposar durante unos minutos y luego poder proceder a medir su pH con el pH metro digital e inmediatamente colocar los resultados obtenidos en la ficha de datos individual. Resultados. En promedio el $\mathrm{pH}$ salival de las personas con diabetes mellitus $(\mathrm{pH}=6.5)$ es menor que el de personas que no poseen la enfermedad $(\mathrm{pH}=8.3)$, lo que nos indica que poseen un $\mathrm{pH}$ salival más ácido. Conclusiones. En promedio el pH salival de las personas diabéticas es menor que las personas sanas, lo que nos indica que poseen un pH salival más ácido; asimismo, los pacientes diabéticos presentan un IHOS en promedio mayor que las personas sanas.
\end{abstract}

Palabras clave: PH salival; Diabetes mellitus; Índice de higiene oral simplificado. (Fuente: DeCS BIREME).

\begin{abstract}
Objective. Determine salivary $\mathrm{pH}$ in compensated adult patients with diabetes mellitus (DM) treated at the Callao public health center. Materials and methods. It was an analytical, observational, case-control study. Sample of 50 people with an age range of 20 to 60 years. A general induction on the study was carried out to obtain informed consent. A general induction on the study was carried out to obtain informed consent. They were instructed to contain the saliva that accumulates in the mouth for a few minutes and then, each minute, the saliva was poured into a glass funnel connected to a clean, disinfected cylinder, graduated in $10 \mathrm{ml}$, this activity was carried out during a minute. Once the sample was collected, it was placed in a cool and safe place to allow it to rest for a few minutes and then be able to proceed to measure its $\mathrm{pH}$ with the digital $\mathrm{pH}$ meter and immediately place the results obtained in the individual data sheet. Results. On average, the salivary $\mathrm{pH}$ of people with diabetes mellitus $(\mathrm{pH}=6.5)$ is lower than that of people who do not have the disease $(\mathrm{pH}$ $=8.3$ ), which indicates that they have a more acidic salivary $\mathrm{pH}$. Conclusions. On average, the salivary $\mathrm{pH}$ of diabetic people is lower than healthy people, which indicates that they have a more acidic salivary pH; likewise, diabetic patients have an OSI higher on average than healthy people.
\end{abstract}

Keywords: Salivary PH; Mellitus diabetes; Simplified oral hygiene index. (Source: MeSH NLM).

\section{INTRODUCCIÓN}

La diabetes es un desafío de salud global que enfrenta el mundo actual. China ocupa el primer lugar con 96.2 millones de individuos que padecen esta enfermedad, luego está la India ocupa el segundo lugar con 66.8 millones. La Federación Internacional de Diabetes estima que, hoy en día, más de 387 millones de individuos a nivel mundial, tienen diabetes y se estima que aumentarán a 592 millones para 2035. Es una enfermedad metabólica crónica común con diversas consecuencias orales. La diabetes mellitus se manifiesta en la alteración de la composición salival y sus funciones. El cambio en el ambiente oral inicia

${ }^{1}$ Universidad Nacional Federico Villareal. Lima-Perú.

a Cirujano Dentista

iD Código ORCID: https://orcid.org/0000-0002-9263-0709 , María Dayan Avellaneda Lopez

Citar como: Avellaneda Lopez MD. Determinación del PH salival en pacientes adultos con diabetes mellitus compensados. Rev Peru Investig Matern Perinat. 2021;10(3): 38-41

DOI https://doi.org/10.33421/inmp.2021241

Recibido: 20-08-2021 Aceptado: 30-09-2021 
bacterias patógenas, dañando los tejidos duros y blandos de la cavidad oral, lo que lleva a un aumento de la actividad cariogénica y lesiones periodontales ${ }^{1}$.

En el Perú, el 3,3\% de la población de 15 a mayores años de edad comunicaron que en algún momento de su vida fueron diagnosticados con diabetes mellitus; este porcentaje se elevó en 0,4 puntos porcentuales en comparación al 2016. La población femenina fue la más perjudicada (3,6\%) en comparación a la masculina $(3,0 \%)$. Asimismo, por región natural, en el 2017 , el porcentaje más elevado de personas con diabetes se encuentran en Lima Metropolitana $(4,1 \%)$ y Resto Costa $(4,0 \%)$ y el porcentaje menor en la Sierra $(1,8 \%)$ y Selva $(2,7 \%)^{2}$.

Recientes evidencias ${ }^{3}$ científicas en investigaciones poblacionales indican que la prevalencia de diabetes mellitus crece y se consignan aproximadamente dos casos nuevos por cada cien individuos anualmente.

Las alteraciones orales usuales, abarcan a la enfermedad periodontal, caries dental, candidiasis, queilitis comisural y sialomegalia. Los anteriores mantienen relación con la xerostomía y la hipofunción glandular. Algunos autores mencionan que la diuresis o poliuria, desencadena una reducción de líquido extracelular, y congruentemente baja producción de saliva ${ }^{4}$.

La diabetes es uno de los problemas más frecuentes que producen alteraciones del sistema estomatognatico. Es un dilema de salud creciente ${ }^{5}$.

El objetivo del presente estudio es determinar el $\mathrm{pH}$ salival en pacientes adultos con diabetes mellitus compensados relacionados a enfermedad de la mucosa bucal en un centro de salud público del Callao.

\section{MATERIALES Y MÉTODOS}

Fue un estudio analítico, observacional, casos y controles. La muestra fue constituida por 50 personas de ambos sexos con diagnóstico de diabetes mellitus compensada cuyo rango en edad fue de 20 a 60 años, que cumplieron con criterios de inclusión: pacientes que hayan tenido la enfermedad por lo menos 5 años, pacientes insulinodependientes, pacientes que hayan asistido al odontólogo los últimos 12 meses a realizarse un control, estar en ayunas y aquellos que aceptaron el consentimiento informado. Fueron excluidos las gestantes y pacientes que recibían hipoglucemiantes orales. Fueron enrolados a partir del 9 hasta el 13 de setiembre 2019 y atendidas en el centro de salud público del Callao.

Procedimientos. Previamente se inició la calibración del pH-metro: el $\mathrm{pH}$ se midió a través de un microprocesador PEN TYPE pH Meter 900 de range: $0.00-14.00 \mathrm{pH}$ marca HENAN.

Una vez estandarizadas las variables se realizó el procedimiento en el consultorio del Centro de Salud del Callao, así mismo se respetaron las normas de bioseguridad básicas como lo indica el Ministerio de Salud en sus normas y procedimientos de atención en la salud bucal.

La técnica se realizó cuando el paciente estuvo en reposo, en ayunas, la estimulación glandular fue mínima y sin estímulos exógenos. Se mantuvo al paciente con la cabeza inclinada hacia delante con los labios entreabiertos. Se les indicó que contengan la saliva que se iba acumulando en la boca por unos minutos y luego, en cada minuto, la saliva se fue vertiendo en un embudo de vidrio conectado a una probeta desinfectada y limpia, graduada en $10 \mathrm{ml}$, esta actividad se realizó durante un minuto. Una vez recogida la muestra se colocó en un lugar fresco y seguro para dejar reposar, y se procedió a medir su $\mathrm{pH}$ con el pH metro digital e inmediatamente se colocó en la ficha de datos. Posteriormente se realizó la examinación del índice de higiene oral simplificado (IHOS) y luego de las superficies dentales, para ello se necesitó pera de aire, una silla cómoda y un equipo de diagnóstico estéril por cada paciente (pinza, espejo y sonda periodontal). Las piezas dentarias que se examinaron se limpiaron con una gasa estéril humedecida con clorhexidina al $12 \%$ y se utilizó un hilo dental en las zonas interdentales (en caso haya sido necesario) todos los datos obtenidos se colocaron en la ficha de datos. Seguidamente se realizó una charla informativa a los pacientes acerca del valor de una correcta higiene oral. Al finalizar el procedimiento, para la eliminación de los desechos contaminados provenientes de la muestra se depositaron en una bolsa de color rojo del mismo centro de salud.

Análisis de datos. Fueron tabulados en el paquete estadístico SPSS 24.0 versión en español y la base de datos se realizaron en un ordenador Intel Core i5, con el sistema operativo Windows 10. Para analizar los datos se usó estadística descriptiva. Antes de cada prueba se estableció si las distribuciones obtenidas son paramétricas $o$ no paramétricas mediante la prueba de Kolmogorov-Smirnov. Se aplicó la prueba de chi cuadrado, se calculó la T de student.

El presente estudio contó con la aprobación de la Dirección de Grados y Títulos de la UNFV y del Comité de Ética de la DIRESA Callao. Se obtuvo consentimiento informado de los participantes; asimismo, se cumplió a cabalidad con los principios planteados en la Declaración de Helsinki en 1975 hasta 2013, referido en proteger la vida y la salud realizadas en los seres humanos durante las investigaciones. 


\section{RESULTADOS}

Tabla 1. Número de personas sanas y con diabetes mellitus de acuerdo al sexo

\begin{tabular}{lcc}
\hline SEXO & DIABÉTICOS & CONTROL \\
\hline Masculino & 10 & 12 \\
\hline Femenino & 15 & 13 \\
\hline
\end{tabular}

En la tabla 1, se observa que la cantidad de pacientes diabéticos del sexo masculino fueron $10(40 \%)$ y $15 \mathrm{del}$ sexo femenino (60\%); mientras del grupo control del sexo masculino fue 12 (48\%) y 13 del sexo femenino (52\%). El promedio de edad de pacientes diabéticos fue 51.32 años y del grupo control 49.44 años.

Tabla 2. Promedio de IHOS de personas sanas y con diabetes mellitus

\begin{tabular}{cc}
\hline Grupo & $\begin{array}{c}\text { Promedio } \\
\text { IHOS }\end{array}$ \\
\hline Diabéticos & 4.11 \\
\hline Control & 1.83 \\
\hline IHOS: índice de higiene oral simplificado
\end{tabular}

La tabla 2, se observa que el promedio del IHOS de pacientes diabéticos fue $4.11 \mathrm{y}$ del grupo control 1.83 .

Tabla 3. Valores del $\mathrm{pH}$ salival en personas sanas y con diabetes mellitus

\begin{tabular}{lcccccc} 
Grupos & N & Media & D.s. & Mediana & Mínimo & Máximo \\
\hline Sanos & 25 & 8.373 & 0.482 & 8.32 & 7.71 & 9.12 \\
\hline Diabéticos & 25 & 6.516 & 0.565 & 6.48 & 5.49 & 7.38 \\
\hline
\end{tabular}

La tabla 3, se observa que el $\mathrm{pH}$ salival en promedio es mayor en pacientes sanos (8.373), los valores obtenidos son mayores de 7 y en personas no sanas el nivel promedio de $\mathrm{pH}$ salival es de 6.516, valores obtenidos de $\mathrm{pH}$ son menores de 7.4.

Tabla 4. Comparación de promedios del nivel de $\mathrm{pH}$ salival entre el grupo de personas sanas y con diabetes

\begin{tabular}{|c|c|c|c|c|c|c|c|c|}
\hline Grupos & N & Media & E.S. & D.s. & \multicolumn{2}{|c|}{ IC $95 \%$} & $t$ & p \\
\hline Sanos & 25 & 8.373 & 0.0964 & 0.4820 & 8.1742 & 85722 & 12.5099 & 0.0000 \\
\hline Diabéticos & 25 & 6.516 & 0.1129 & 0.5645 & 6.2830 & 6.7490 & & \\
\hline
\end{tabular}

La tabla 4, se observa que los niveles de $\mathrm{pH}$ salival difieren entre las personas sanas y los pacientes diabéticos, esta diferencia es estadísticamente significativa.
Tabla 5. Correlación de medias del nivel de $\mathrm{pH}$ salival entre el grupo de personas sanas y con diabetes

\begin{tabular}{lcccccc}
\hline Personas & \multicolumn{3}{c}{ Sanas } & \multicolumn{3}{c}{ Diabéticos } \\
\hline Factores & $\mathrm{N}^{\circ}$ & $\mathrm{R}$ & $\mathrm{P}$ & $\mathrm{N}^{\circ}$ & $\mathrm{R}$ & $\mathrm{P}$ \\
IHOS & 25 & -0.7055 & $0.0001^{*}$ & 25 & -0.9055 & $0.0000^{*}$ \\
Edad & 25 & -0.3995 & $0.0479^{*}$ & 25 & -0.6357 & $0.0006^{*}$ \\
Tiempo & 25 & - & - & 25 & -0.0606 & 0.7737 \\
\hline
\end{tabular}

En la tabla 5, se observa que, tanto en el grupo sano como en diabéticos, existe una correlación negativa significativa entre IHOS y edad con el nivel de $\mathrm{pH}$ salival; también a medida que elevan la edad y el IHOS, el nivel de $\mathrm{pH}$ disminuye significativamente $(\mathrm{p}>$ 0.05).

\section{DISCUSIÓN}

Se observó que en promedio el $\mathrm{pH}$ salival de las personas diabéticas es menor que las personas sanas, lo que nos indica que poseen un pH salival más ácido. Al comparar el nivel de $\mathrm{pH}$ salival entre el conjunto de personas sanas y el colectivo de personas con diabetes mellitus, se encontró que si hay diferencia estadística entre ellos. Asimismo, se encontró que estadísticamente es significativo el coeficiente de la variable IHOS como factor de riesgo influyente en el $\mathrm{pH}$ salival entre personas sanas y con diabetes mellitus. Según reportan Seethalakshmin $\mathrm{C}$ et al ${ }^{1}$ que hay una relación significativa entre la diabetes mellitus y el aumento de la incidencia de caries dental y periodontitis y también una reducción significativa del pH salival en sujetos con diabetes mellitus, en comparación con la de sujetos sanos. Dicho resultado tiene concordancia con esta investigación ya que los valores obtenidos de la muestra se asemejan a los datos encontrados.

Asimismo, según reportes de Carda $\mathrm{C}$ et al ${ }^{4}$ las manifestaciones de xerostomía se localizaron en el $76,4 \%$ de los casos y las lesiones dentales y periodontales en el $100 \%$ de los pacientes; por lo que concluyeron que las alteraciones de carácter bioquímico en la saliva de los diabéticos tipo 2 se pueden correlacionar con las alteraciones estructurales. Dicho resultado tiene concordancia con esta investigación, ya que a menor nivel de $\mathrm{pH}$ salival en pacientes con diabetes existen mayores alteraciones en la cavidad bucodental.

En conclusión, en promedio el pH salival de las personas diabéticas es menor que las personas sanas, lo que nos indica que poseen un pH salival más ácido; asimismo, los pacientes diabéticos presentan un IHOS en promedio mayor que las personas sanas.

Declaración de conflictos de intereses: La autora declara no tener conflicto de intereses.

Financiamiento: Autofinanciado. 


\section{REFERENCIAS BIBLIOGRÁFICAS}

1. Seethalakshmi C, Jagat R, Asifa N, Prabhu S. Correlación del ph salival, incidencia de caries dental y estado periodontal en pacientes con diabetes mellitus: un estudio transversal. Journal of clinical y diagnostic research. 2016; 10(3): ZC12-ZC14.

2. Instituto Nacional de Estadística e Informática. Enfermedades no transmisibles y transmisibles. Perú 2018. Recuperado de https://www.inei.gob.pe/media/MenuRecursivo/ publicaciones_digitales/Est/Lib1526/libro.pdf

3. Carrillo R, Bernabé A. Diabetes mellitus tipo 2 en Perú: Una revisión sistemática sobre la prevalencia e incidencia en población general. Rev. Perú Med Exp Salud Publica.2019; 36(1): 26-36.

4. Carda C, Mosquera-Lloreda N, Salom L, Gomez de Ferraris ME, Peydró A. Structural and functional salivary disorders in type 2 diabetic patients. Med Oral Patol Oral Cir Bucal 2006;11:E309-14

5. Hechavarria B, Nuñez L. La diabetes mellitus y sus manifestaciones bucales. KIRU.2017; 14(2): 193-197.

\section{Correspondencia:}

María Dayan Avellaneda Lopez

Dirección:

Correo: mariadayanavellaneda@gmail.com

Teléfono: 948140233 